\title{
Establishing an Innovation-Oriented National Preventive Medicine System in China
}

\author{
Xu Jianguo ${ }^{1}$, Liu Kaitai ${ }^{2}$, Chen Bowen ${ }^{3}$, Jia Guang ${ }^{4}$, Shao Ruitai ${ }^{5}$, Yin Delu ${ }^{6}$, Yin Jiyong ${ }^{2}$, Xue Dongmei ${ }^{1}$, Hu \\ Guiping $^{4}$, Ma Jun ${ }^{4}$, Sun Changhao ${ }^{7}$, He Yanling ${ }^{8}$, He Yao ${ }^{9}$, Li Liping ${ }^{10}$, Yang Kedi ${ }^{11}$, Liang Hong ${ }^{12}$, Guo Youde ${ }^{12}$, \\ Wen Chunmei $^{13}$, Han Biao ${ }^{1}$, Wu Yangfeng ${ }^{14}$, Dai Zheng ${ }^{15}$
}

1. National Institute for Communicable Disease Control and Prevention, Chinese Center for Disease Control and Prevention, Beijing 102206, China

2. National Institute for Nutrition and Health, Chinese Center for Disease Control and Prevention, Beijing 100050, China

3. Community Health Association of China, Beijing 100020, China

4. School of Public Health, Peking University, Beijing 100191, China

5. World Health Organization, Geneva 1121, Switzerland

6. Capital Institute of Pediatrics, Beijing 100020, China

7. Public Health College of Harbin Medical University, Harbin 150081, China

8. Shanghai Jiao Tong University School of Medicine, Shanghai 200025, China

9. Institute of Gerontology, Chinese PLA General Hospital, Beijing 100853, China

10. Shantou University Medical College, Shantou 515063, Guangdong, China

11. School of Public Health, Tongji Medical College, Wuhan 430074, China

12. School of Social Development and Public Policy, Fudan University, Shanghai 200433, China

13. WHO Representative Office in China, Beijing 100600, China

14. Peking University Clinical Research Institute, Beijing 100191, China

15. Education and Training Division, Chinese Center for Disease Control and Prevention, Beijing 102206, China

\begin{abstract}
With China's economic development and lifestyle changes, the spectrum of diseases and risk factors affecting people's health has drastically changed. The current systems including health laws and decrees, education about clinical and preventive medicine, health services, and disease prevention and control do not adequately promote and protect the health of Chinese people. In this paper, we analyze China's historical achievements as well as current challenges and opportunities in healthcare policy formulation and implementation, and administrative strategy. We discuss how to establish an innovation-oriented national preventive medicine system to make efficient and maximum use of limited resources to control the rapid increase in the prevalence of chronic noncommunicable diseases and to protect and promote optimum public health across the nation.
\end{abstract}

Keywords: public health; preventive medicine; disease prevention and control; noncommunicable diseases; health risk factors; strategy

\section{Introduction}

The Chinese government has implemented the policy of prioritizing prevention in medical administration and services since the foundation of the People's Republic of China. Controlling infectious, endemic, and noncommunicable (chronic) diseases that affect residents' lives and health has been the focus of health work. Health services in urban and rural areas have been

Received date: January 10, 2017; Revised date: February 10, 2017

Corresponding author: Xu Jianguo, National Institute for Communicable Disease Control and Prevention, Chinese Center for Disease Control and Prevention, Researcher; Chinese Academy of Engineering, Academician. Major research fields are medical microbiology and new emergent infectious diseases. E-mail: xujianguo@icdc.cn

Funding program: CAE Advisory Project “Development Strategy for National Health Promotion and Medical and Healthcare Undertakings in China” (2014-ZD-06) Chinese version: Strategic Study of CAE 2017, 19 (2): 055-061

Cited item: Xu Jianguo et al. Establishing an Innovation-Oriented National Preventive Medicine System in China. Strategic Study of CAE, https://doi.org/10.15302/ J-SSCAE-2017.02.009 
strengthened. In fact, the prevention and control of major diseases have become more effective. For example, maternal and child health policies have been effectively implemented, leading to a significant decrease in infant and maternal mortality rates. Moreover, disease prevention and control in environmental, occupational, radiological, school, and nutritional health have been continuously developed, resulting in great practical achievements. Effective prevention and control strategies have also been applied to public health emergencies such as Ebola, severe acute respiratory syndrome (SARS), and post-earthquake epidemic situations. In addition, community health services conveniently provide daily health services and promote the specific measures applied in disease prevention and control [1,2].

In recent years, the main disease spectrum, health risk factors, and social conditions affecting people's health in China have drastically changed. China is facing both the threat of emergence or resurgence of known or unknown infectious diseases and the high incidence of chronic diseases. The health service and education systems were established primarily to treat infectious and acute diseases. However, in the present scenario, they have proved insufficient to manage the serious outbreak of chronic diseases in terms of meeting treatment requirements as well as combating financial challenges. China needs a comprehensive national health law to effectively protect and promote health [3-5]. During the China National Health and Wellness Conference held on August 19 and 20, 2016, Communist Party of China General Secretary Xi Jinping stressed the importance of prevention, which is the most economical and effective health strategy.

This paper aims to analyze the major preventable and controllable diseases and public health problems prevalent amongst the Chinese population, explore the methods to establish an innovation-oriented national preventive medicine system by adapting to practical demands, and address the importance of a national health law and practicable administrative policies to maximally use limited resources and optimize the role of public organizations in prevention and promotion of health.

\section{Achievements of health work in China}

After decades of effort, China has made great progress in disease control and prevention. The main achievements are: good control of common contagious diseases through implementation of the prevention and control measures set in place by the sanitation and epidemic control organs according to the Law on Prevention and Cure of Contagious Diseases; establishment of a nationwide three-level health service network and emergency response mechanism for infectious diseases for both urban and rural areas; focus of health care work on rural areas to effectively protect the majority of the vulnerable population; nutritional intervention, immunization programs, and chronic disease management for pregnant and lying-in women, children, and other vulnerable target populations; and carrying out prevention and control measures, with significant and effective protective roles, for childhood pneumonia, hepatitis B, tuberculosis, schistosomiasis, and key endemic diseases [1,2]. Primary and community health care services have promoted the more rational application of drugs and technologies, making it more convenient and accessible for community residents to get immediate treatment for common diseases such as colds, pneumonia, and influenza. The incidence of notifiable infectious disease in China was reduced by $93.2 \%$, from $7000 / 100000$ in 1970 to $473.9 / 100000$ in 2013 . The average life expectancy of Chinese residents increased from 35 to 76 between 1950 and 2010 [6].

\section{Prevalent diseases and public health problems in China}

The current focal point of health work in China is analyzing health risk factors, assessing disease patterns, and implementing effective prevention and control measures to further promote people's health.

3.1 Some traditional serious infectious diseases like hepatitis and tuberculosis have not been controlled thoroughly

Owing to the state of the global economy, trade development, and constant increase in international cooperation and exchanges, new and unknown infectious diseases have become a major challenge to global public health, significantly affecting social and economic stability and security. In recent years, it has so happened that bringing infectious diseases under control has been followed by the emergence of new infectious diseases. The outbreaks of Ebola and SARS had greatly threatened global health and survival, causing international concern and panic, forcing the world to mobilize and invest in a lot of human, financial, and material resources to control the spread of these diseases [7].

3.2 Chronic diseases have become the leading cause of death among Chinese residents

With environmental pollution, unhealthy diets, a lack of physical exercise and health knowledge, and so on, chronic diseases such as high blood pressure, diabetes, coronary heart disease, stroke, and tumor have become the leading cause of deathaccounting for more than $80 \%$ of all deaths-among Chinese residents. About three million people die prematurely each year $[8,9]$. The primary reason is that the current system has not been effective in actively preventing and controlling chronic diseases. From the professional perspective, the gap between clinical and preventive medicine has deepened. Regarding administration, government departments lack a thorough understanding of disease prevention and control, with each working individually 
and seldom coordinating well. For example, less than $30 \%$ of disease control and prevention organizations all over the country monitor risk factors for chronic diseases [10]. It is necessary to encourage government departments and various clinical and preventive medicine professionals to achieve the final goal of improving the physical quality and fitness of the whole population by mobilizing efforts to improve the executive ability.

\subsection{Mental and psychological problems are increasing drastically}

In this period of rapid economic development and transformation, the occurrence and drastic increase of psychiatric diseases is a common phenomenon. The World Health Organization has predicted that by 2020, China's neuropsychiatric burden will be up to a quarter of the country's total disease burden $[11,12]$. This is a cause for concern because, in the present scenario, China's experts, doctors, mental health intervention staff, and medical institutions are not fully equipped to treat psychiatric diseases in China.

\subsection{Occupational, environmental, and other public health issues need to be considered}

Occupational hazards have not yet been fundamentally controlled in China [13]. The quality of water, air, soil, and other important environmental factors closely related to people's daily life has deteriorated [14-16]. The number of smokers in China is more than 300 million, with more than one million people dying of tobacco-related causes each year $[17,18]$. The rates of common diseases such as obesity, myopia, and other related issues among children and adolescents are high. If the basic environmental factors influencing these epidemics are not radically improved, China will not be able to maintain and improve people's health in the short term and achieve the long-term goal of a healthy population.

\subsection{Aging of the population aggravates the burden of disease}

With China's rapidly aging population, the increase in corresponding health care burden is inevitable. If effective disease control strategies are not implemented to maintain the elderly population's health, the overlapping burdens of chronic diseases and health service in the aging society will increase significantly [19].

China is facing multiple interrelated challenges of infectious diseases, chronic diseases, mental disorders, and environmental degradation. Changes in disease patterns and control are closely related to socioeconomic development, unhealthy behavior, environmental pollution, and population aging [20]. Therefore, in this present situation, it is imperative to establish optimized health policies and legislation systems and enhance the protection of nationwide health.

\section{The current gap and challenges in China's health system}

\author{
4.1 Policymakers and the public lack adequate \\ understanding of the seriousness of disease and health \\ problems and the related coping strategies
}

One of the basic elements of a civilized and harmonious society is maintaining a conscious, healthy, and vibrant population. Owing to a history of poverty, China has strongly focused on economic and social development to ensure people achieve a basic well-off life. Although economic and social development has helped meet basic living and health needs, it has led to negative effects such as environmental degradation, accelerated disease transmission, and unhealthy lifestyles, resulting in epidemics of some preventable and controllable diseases. Health literacy in China is limited, and instead of making good choices and taking the necessary actions to maintain their health, people assume that doctors and hospitals are responsible for their health protection.

\subsection{The existing medical care system and education are insufficient for the prevention and control of key public health issues and diseases}

China's existing medical care, public health, and preventive medicine education systems, developed based on the notion of epidemics of biomedical infectious diseases and the treatment of acute diseases, lack the expertise and infrastructure required for the prevention and control of noncommunicable diseases, which are caused by biological, occupational (environmental), behavioral, and social factors. Developed for the prevention of infectious diseases, existing systems are unequipped to conduct early screening, health counseling, and long-term follow-up and treatment, all of which are necessary for preventing and controlling chronic diseases [21].

\subsection{Government investment in medical and health fields is improper with an unreasonable distribution of medical resources}

China's financial investment in health care lacks rigorous technical audit and corresponding laws, regulations, policy support, and restrictions. Investment in health has been inclined toward the construction of hospitals, most of which are in large, central cities. Owing to traffic inconveniences and most people's inability to pay large sums for health care, only a few can use these high-tech facilities, while the majority of the communities in rural areas lack access to basic medical and health services. Such big financial investments in large hospitals have greatly increased the cost of medical services. At the same time, they compete with grassroots and community health services for lim- 
ited resources. Resultantly, it has become more difficult for basic medical and community health services to receive basic investment and protection, leading to a vicious cycle of people from rural communities lacking medical care and support measures for chronic disease treatment.

\subsection{The increase in the gap between clinical medicine and public health}

The interrelation between clinical and preventive medicine education needs more attention. In practice, the cooperation between preventive and clinical medicine should be strengthened, especially for the prevention and treatment of chronic diseases. Advanced clinical technology such as early recognition and treatment of chronic diseases needs to be promoted and applied in centers for disease control. It is not ideal to depend on doctors for chronic disease control because clinical treatment focuses on individual diseases and ignores the epidemiology of chronic diseases. Thus, it does not thoroughly control the incidence of diseases. The increasing gap and inconsistencies between clinical and preventive medicine may lead to a distancing from their mission to protect and guarantee people's health [22].

\subsection{Existing health information systems cannot fully reflect the health status of residents}

In comparison to developed countries, there is a big lag in China's health statistical information, especially regarding death causes and other key statistics. Therefore, data on people's life process is incomplete. It is not possible for the government to make optimal health decisions when the concerned information is not accurate or up to date.

\subsection{The health decision-making mechanism is not ideal}

Health is a basic need; so, as a society, we need to continuously seek to improve the level of health care. Nowadays, China's health decision-making method is makeshift and sector-based and uses outdated and time-consuming decisionmaking strategies. Health decision-making procedures and mechanisms lack a scientific, transparent, integrated, macro, and long-term perspective. An important way to achieve national health and develop a healthy Chinese population is to mobilize the masses to participate and address innovation. The major reform initiative currently being undertaken by Chinese health services is to improve the quality of disease prevention and control.

\subsection{Lack of supervision and long-term follow-up assessment mechanism; decoupling of performance and health effects}

The visible results of financial investment and lack of planning such as in hospitals and equipment could lead the govern- ment to focus on short-term investment for prompt performance assessment, ignoring areas like grassroots community health services in need of long-term investment. The lack of a scientific follow-up assessment mechanism will extensively accelerate this vicious circle.

\section{Mission of public health undertakings}

The mission for disease prevention and the public health system that we recommend aims to reinforce early detection of emerging infectious diseases, strengthen surveillance and emergency response capabilities, reduce premature death caused by chronic diseases, improve health-related quality of life, and build a harmonious and healthy aging society.

The specific objectives are to (1) establish public health service agencies and a flawless medical education system providing proper preventive and clinical medicine training to professionals; (2) organize a strong professional network with capabilities for crisis response, strengthening operational readiness for new infectious diseases within high-tech conditions; and (3) strengthen basic community health services and capacity building to promote the use of new technology, material, and techniques for controlling chronic diseases and mental health issues in communities across the nation. The development strategies should combine prevention with treatment, strengthen international and domestic coordination, and reinforce sectoral coordination and cooperation, amongst other things.

\section{Suggestions}

\subsection{To identify diseases and issues affecting public health}

Owing to China's large population, unbalanced development, the double burden of infectious and chronic diseases, and coexistence of poverty and wealth-related diseases, it is difficult to coordinate the management of all diseases and health problems at a national level. Therefore, it is necessary to refer to international best practices and identify priorities for strengthening green management, carrying out clean production, improving occupational conditions and enhancing related disease prevention and control, strictly controlling health risk factors, optimizing living conditions, and creating a healthy environment to achieve primary prevention for common diseases and issues affecting public health.

\subsection{To put health protection into all policies}

6.2.1 Health protection and socioeconomic development should be equally important

Major diseases and important public health problems can affect socioeconomic development, social stability, and public health. Experiences in developed countries have shown that the 
control of major diseases must be part of strategies for socioeconomic development. Health protection needs to be ensured by a national health law, together with sufficient financial investment and other social funds. This will help guarantee economically and socially sustainable development, develop a comprehensive well-off and healthy aging society, and make the realization of China's dream possible.

\subsubsection{Develop a national health law}

It is necessary to speed up the process of developing a fundamental health law with top-level design to coordinate the implementation of other policies and regulations. The national health law will benefit organizational consolidation, ideological education, and rectification of work style related to health protection and promotion.

6.2.3 Build up level-by-level responsibility of preventive health care funding

The roles of the respective government departments are further defined hereunder. The central government is fully responsible for the preventive health care costs of grassroots communities (neighborhoods) and the neighborhood committees (village committees). Provincial, municipal, and county governments are responsible for preventive health care costs at provincial, city, and district levels. The payment model that is used to buy prevention and health care services needs to be improved. This can be done by encouraging social funds to donate to disease prevention, as well as the market and the society as a whole to use their advantage to further enhance the efficiency and coverage of health care.

6.2.4 Establish special funding for prevention and control of high-prevalence chronic diseases

An increase in funding dedicated to severe chronic diseases such as hypertension and diabetes as well as preventable major diseases is necessary. Some major disease prevention and control demonstration sites should be established. Further, more effective methods for prevention and control need to be summed up and popularized.

6.2.5 Build a healthy environment and promote the transformation of scientific and technological achievements

Publicity about health strategies and the policy of promoting the health of the people should be increased. The establishment of a more suitable health education system, popularization of the knowledge of health science, advocating the concept that everyone is responsible for their own health, guiding a healthy and civilized lifestyle, educating people in the right way to approach health, and strengthening the general cognition about prevention and treatment of chronic diseases are suggested. The government should supply policy support, increase investment, and carry out early disease screening and detection so as to achieve prima- ry and secondary prevention for most diseases. Moreover, we should enhance positive publicity, public opinion supervision, scientific guidance, and make full use of mainstream and new media to carry out various forms of chronic disease prevention and control. In addition, it is proposed to carry out systematic scientific research for chronic disease prevention and control, promotion of related scientific research projects, and transformation and application of appropriate scientific and technological achievements.

\subsection{To develop a public health system that adapts to the practical demands of disease prevention and control}

To prevent and control serious diseases and solve the problem of public health, we should be equipped with high-level technology and have the capability to provide national service with extensive coverage and fast response, as well as have a public health network and service system that can work internationally. This includes the following aspects:

6.3.1 Strengthening international and domestic coordination and upgrading overall response and control abilities focusing on both known and unknown infectious diseases

New control strategies should pay more attention to early detection and recognition of infectious diseases, whilst closely cooperating with international society to control epidemic outbreaks. Simultaneously, it is necessary to strengthen communication and coordination among relevant health, transportation, customs, tourism, commerce, and public security departments to implement control measures according to the law to restrain transmission. Moreover, the ability of technical analysis and epidemiological investigation needs to be improved.

6.3.2 The responsibilities of China's disease prevention and control centers should be allocated to fit the context

China's disease prevention and control system should pay attention to both ends, with one end being addressed by primary or secondary prevention incentives. Specialized national and provincial prevention agencies need to strengthen scientific research, draw up national guidelines, and develop appropriate health techniques and services. The other part needs to focus on the implementation of services in grassroots areas hospitals and community health centers, township health centers, or village health rooms.

The grassroots disease prevention and control organizations need to be integrated with communities' primary clinical hospitals to improve early detection and prevention, technical guidance, and monitoring capabilities in the context of infectious and chronic diseases. Further, it is necessary for superior disease prevention and control organizations to readjust functions within the rapid coordination of and response capabilities toward infectious diseases, while developing chronic disease control and 
treatment efficient techniques and providing technical support of other public health issues.

6.3.3 Demand-oriented training for public health professionals

It is suggested that a reasonable public health professionals' course for students with specialized training in clinical practice, sociology, psychology, biology, politics, economics, and other related topics be set up. In this way, China can cultivate senior public health professionals with sufficient academic backgrounds of a master's degree or above. Public health practitioners need to have prescription rights and diagnosis and disease treatment capabilities, which should be included in the physician assessment management system.

In conclusion, China's economic development and the improvement of people's living conditions have brought unprecedented opportunities and challenges to the development of preventive medicine. The current public health service system needs further reforms, opening wider internationally to meet the strong demands of Chinese people's health protection and promotion. Developing an overall well-off society cannot be realized without a modern public health system specifically adapted to the Chinese population.

\section{References}

[1] Li L M, Jiang Q W. Theory and practice of public health in China [M]. Bejing: People's Medical Publishing House, 2015.

[2] Li L M. Achievements and prospects of public health in sixty years in New China [J]. Chinese Journal of Public Health Management, 2014, 30(1): I0001-I0002.

[3] Meng Q Y, Yang H W, Chen W, et al. People's Republic of China health system review [J]. Health Systems in Transition, 2015, 5(7):1-142.

[4] Han Qide. China's Health Development Is Still Facing Serious Challenges [J]. Chinese Health Policy Research, 2010(5): 41.

[5] Li T, Wang X F. Connotation and realizing route of healthy China [J]. Health Economics Research, 2016, 345(1): 4-9.

[6] Ministry of Health Statistical Information Center. China health statistics yearbook in 2013 [Z]. Beijing: Pecking Union Medical College Press, 2013.

[7] Gong Lei, Zhang Jin, Chen Guoping, et al. A review on epidemic and early recognition and warning of emerging infectious diseases [J]. Anhui Journal of Preventive Medicine, 2015, 21(2): 117-121.
[8] Listed N. China's major health challenge: Control of chronic diseases [J]. Lancet, 2011, 378(9790): 457.

[9] Wei W. 3 million people die prematurely each year in China due to chronic diseases [J]. Family Medicine, 2015(2): 32.

[10] Si X, Zhai Y, Shi X M. Assessment on the capacity for programs regarding chronic non-communicable diseases prevention and control in China [J]. Chinese Journal of Epidemiology, 2014, 35(6): 675-679.

[11] Alonso J, Chatterji S, He Y. The burden of mental disorders: Global perspectives from the WHO world mental health surveys [M]. New York: Cambridge University Press, 2013.

[12] Phillips M R, Zhang J, Shi Q, et al. Prevalence, treatment, and associated disability of mental disorders in four provinces in China during 2001-05: An epidemiological survey [J]. Lancet, 2009, 373(9680): 2041-2053.

[13] Zhang X. Elimination of silicosis has a long way to go [J]. Chinese Journal of Industrial Hygiene and Occupational Diseases, 2012 30(1): $1-2$.

[14] Jia G. Healthy China, occupational health first [J]. Journal of Peking University (Health Sciences), 2016, 48(3): 389-391.

[15] Pan X C. Pay attention to Chinese atmospheric haze (PM2.5) new normalization effects on human health [J]. Journal of Peking University (Health Sciences), 2015, 47(3): 377-379.

[16] Du G. Present situation and countermeasures analysis of China's environmental protection in the new period [J]. Resource Conservation and Environmental Protection, 2015(4): 136-148.

[17] Li S, Meng L, Chiolero A, et al. Trends in smoking prevalence and attributable mortality in China, 1991-2011 [J]. Preventive Medicine, 2016(93): 82-87.

[18] Gu D, Kelly T N, Wu X, et al. Mortality attributable to smoking in China [J]. New England Journal of Medicine, 2009, 360(2) 150-159.

[19] Du P, Zhai Z W, Chen W. The elderly population of China: A century-long projection [J]. Population Research, 2005, 29(6): 92-95.

[20] Chen X S, Gao R L. Persevere in reform and innovation, promote the development of healthy China [J]. National Medical Journal of China, 2017, 97(1): 1-2.

[21] Si X, Zhai Y, Shi X M. Assessment on the capacity for programs regarding chronic non-communicable diseases prevention and control in China [J]. Chinese Journal of Epidemiology, 2014, 35(6): 675-679.

[22] Huang J H. To explore the preventive medicine and clinical medicine development $[\mathrm{J}]$. Chinese and Foreign Medical Research, 2012, 10(5): 154 . 